\title{
SYSTEMIC TREATMENT IN SEVERE CASES OF RECURRENT APHTHOUS STOMATITIS: AN OPEN TRIAL
}

\author{
Maria Angela Martins Mimura, ${ }^{\mathrm{I}}$ Silvio Kenji Hirota, ${ }^{\mathrm{I}}$ Norberto Nobuo Sugaya, ${ }^{\mathrm{I}}$

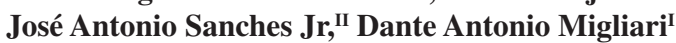

doi: 10.1590/S1807-59322009000300008

\begin{abstract}
Mimura MAM, Hirota SK, Sugaya NN, Sanches Jr. JA, Migliari DA. Systemic treatment in severe cases of recurrent aphthous stomatitis: an open trial. Clinics. 2009;64(3):193-8.
\end{abstract}

PURPOSE: This study aimed to evaluate the efficacy of the systemic drugs thalidomide, dapsone, colchicine, and pentoxifylline in the treatment of severe manifestations of RAS.

METHODS: An open, 4-year clinical trial was carried out for 21 consecutive patients with severe RAS. Initially, patients were given a 2-week course of prednisone to bring them to a baseline status. Simultaneously, one of the four test drugs was assigned to each patient to be taken for a period of 6 months. During the course of the trial, patients were switched to one of the other three drugs whenever side effects or a lack of satisfactory results occurred, and the 6-month limit of the treatment was then reset.

RESULTS: The most efficient and best-tolerated drug was thalidomide, which was administered to a total of eight patients and resulted in complete remission in seven (87.5\%). Dapsone was prescribed for a total of nine patients, of whom eight (89\%) showed improvement in their symptoms, while five showed complete remission. Colchicine was administered to a total of ten patients, with benefits observed in nine (90\%), of whom four showed complete remission. Pentoxyfilline was administered to a total of five patients, with benefits observed in three (60\%), of whom one patient showed complete remission.

CONCLUSION: The therapeutic methods used in this trial provided significant symptom relief. Patients experienced relapses of the lesions; however, this occurred after withdrawal of their medication during the follow-up period.

KEYWORDS: Aphthous stomatitis; Thalidomide; Colchicine; Pentoxyfilline; Dapsone.

\section{INTRODUCTION}

Recurrent aphthous stomatitis (RAS) is a common oral inflammatory disease characterized by painful recurrent ulcerations of the oral mucosa. ${ }^{1-3}$ RAS shows no predilection for sex, and it seems to be more common among white

\footnotetext{
IDepartment of Stomatology, School of Dentistry, University of São Paulo - São Paulo/SP, Brazil.

IIDivision of Dermatology, Hospital das Clínicas, Faculdade de Medicina da Universidade de São Paulo - São Paulo/SP, Brazil.

Tel.: 55113091.7883

Email: nnsugaya@usp.br

Received for publication on September 12, 2008

Accepted for publication on November 21, 2008
}

individuals. There is some evidence that the disease has a higher prevalence in younger adults, decreasing in both incidence and severity with age. ${ }^{2-5}$ The cause of RAS remains unknown, but there is growing evidence for an immunogenic basis for its development. $3,6,7$

RAS occurs in three clinical presentations: minor, major, and herpetiform. Minor RAS is the most common form, and it is characterized by shallow, well-defined ulcers of less than $1 \mathrm{~cm}$ in diameter surrounded by an erythematous halo. These ulcers heal in 7-14 days without scarring. Major RAS is less common, but more severe than the minor form. The ulcers are larger and deeper, with a raised irregular border, and they frequently exceed $1 \mathrm{~cm}$ in size. Major RAS can last for weeks to months, and usually heals with scarring. 
Herpetiform RAS is the rarest form of the disease, and it manifests as clusters of 10-100 small ulcers of 1-3 mm in diameter, which tend to coalesce into large ulcers and follow a clinical course similar to that of minor RAS (healing without scarring). ${ }^{1,8-10}$

A diagnosis of RAS is almost invariably established on the basis of the patient's clinical history and presentation of the lesions. ${ }^{1,2}$ Although histological examination is not in itself sufficient for a definitive diagnosis of RAS, it can aid in the differential diagnosis by eliminating other diseases that may resemble RAS clinically, including erosive oral lichen planus, vesiculobullous diseases, and oral cancer. Herpetiform RAS can be distinguished from intra-oral herpetic lesions via exfoliative cytology techniques and clinical features (e.g., herpetic, recurrent oral lesions usually affect keratinized oral mucosa, the gingival or hard palate areas, and are preceded by vesicles). A detailed clinical history is also important in each case of RAS to exclude other diseases that can present aphthouslike lesions but cannot be differentiated from RAS based on histology. These include Behcet's syndrome, Crohn's disease, human immunodeficiency virus (HIV) infection, and neutropenia. ${ }^{1,10-13}$

There have been many attempts over the years to find an effective treatment for RAS. Since the etiology of RAS remains unknown, its treatment consists of therapeutic measures to suppress the symptoms rather than bringing about a definitive cure. The therapeutic choice depends on the severity of the disease, including the frequency of ulcer recurrence, the number of ulcers present, their location and duration, and the level of associated orofacial pain. Patients with light-to-moderate symptoms and infrequent ulcers may only require palliative therapy for pain. ${ }^{8}$ Cases of severe RAS, those with painful ulcers, and those that show a high frequency of recurrence usually require systemic therapy. The use of immunosuppressive and anti-inflammatory drugs has demonstrated varying degrees of success in severe cases of RAS. Drugs commonly used include corticosteroids, dapsone, colchicine, thalidomide, pentoxifylline, low-dose interferon- $\alpha$, and levamisole. ${ }^{14-17}$

The purpose of the present investigation was to evaluate the clinical response to systemic drugs in the treatment of patients with severe RAS.

\section{METHODS}

Patient selection. Patients were selected for this study based on a severe clinical course of RAS, i.e., with multiple episodes of ulcers monthly. Patients with any hematologic disease, Behcet's syndrome, Crohn's disease, HIV infection or Reiter's syndrome, either initially or as a later development, were not included in the study. Patients whose oral ulcerations could be associated with a drug reaction were also excluded.

Diagnosis of RAS. Diagnosis was made based on the clinical presentation of lesions, as described in the Introduction. Histological and direct immunofluorescence examination was available when the clinical diagnosis was uncertain and was used mainly for differentiation from vesiculobullous diseases. A blood-cell count with determination of hemoglobin concentration, coupled with measurements of glucose-6-phosphate dehydrogenase (G6PD), glycemia, and liver enzyme levels was performed before the trial and twice a month during treatment for all patients.

Patients. During the period between 2002 and 2005, 32 consecutive patients were referred to the Clinic of Oral Diagnosis, Division of Dermatology, University of São Paulo, and subsequently diagnosed as having severe clinical manifestations of RAS. Later, five of the patients were found to have one of the aforementioned systemic diseases (one patient with HIV infection, one with leukemia, two with anemia, and one with Behcet's syndrome), and thus their data was excluded from the study. The remaining 27 patients were enrolled in the treatment protocol. This study was approved by the hospital's Committee on Ethics, and informed consent was obtained from all participants.

Treatment protocol. Before entering the study, patients were informed about the trial, the medications and their possible side effects. A clinical history concerning the ulcer type, size, number, recurrence, healing time, and symptoms were recorded for each patient. Initially, patients were given systemic prednisone for a 2 -week period, starting with $0.5 \mathrm{mg} / \mathrm{kg} /$ day as a single dose in the morning, which was reduced after 1 week to half the initial dose. Simultaneously, one of the four test drugs - namely thalidomide, dapsone, colchicine, or pentoxifylline - was assigned to each patient, keeping the proportion of assignments as equal as possible under the conditions that thalidomide was not prescribed for fertile women, while dapsone was not prescribed for patients with low levels of G6PD and pentoxifylline was only prescribed for patients who could afford it, since it was the only drug that could not be supplied free of charge by the hospital. After withdrawing the prednisone, the assigned drug was maintained for 6 months, unless adverse side effects or unsatisfactory results occurred. In either of these cases, the patient was switched to one of the other three drugs following the same safety criteria described above. The drugs and dosages employed are shown in table 1.

Evaluation and follow-up. Patients were evaluated at 15day intervals during the period of medication usage. At each visit, data regarding clinical status (benefits or side effects) 
Table 1 - Therapeutic schedule

\begin{tabular}{ll}
\hline Drugs & Dosage \\
\hline $\begin{array}{l}\text { Prednisone } \\
\text { Byyny }{ }^{[33]}\end{array}$ & $\begin{array}{l}0.5 \mathrm{mg} / \mathrm{kg} / \text { day for } 7 \text { days } \\
\text { half of the dose for } 7 \text { days }\end{array}$ \\
\hline $\begin{array}{l}\text { Thalidomide } \\
\text { Bonnetblanc et al. }{ }^{[29]}\end{array}$ & $100 \mathrm{mg} /$ day \\
\hline Dapsone & $25 \mathrm{mg} /$ day for 3 days \\
Ahmed et al. ${ }^{[30]}$ & $50 \mathrm{mg} /$ day for 3 days \\
& $75 \mathrm{mg} /$ day for 3 days \\
\hline Colchicine & $100 \mathrm{mg} /$ day maintenance dose \\
Ruah et al. ${ }^{[11]}$ & $0.5 \mathrm{mg} /$ day for 7 days \\
\hline Pentoxifylline & $1.0 \mathrm{mg} /$ day for 7 days \\
Pizarro et al. ${ }^{[22]}$ & $1.5 \mathrm{mg} /$ day maintenance dose \\
\hline
\end{tabular}

were recorded, as well as compliance with the treatment. Any time that a drug had to be replaced with an alternative drug, the 6-month time limit for using the medication was reset. The criteria for drug efficacy were mainly based on the potential to prevent relapses and/or reduce symptoms, numbers of ulcers, and healing time. Drug efficacy was classified as excellent, moderate, mild, or no response as follows: excellent - patient with no relapses; moderate patient showing relapses but with decreasing frequency, less lesions per cycle, and alleviated symptoms; mild - relief of symptoms only; no response - no improvement observed or reported. After withdrawal of the medication, patients were followed for a period ranging from 6 to 12 months.

\section{RESULTS}

Of the 27 patients, six stopped showing up for treatment, and the data for these individuals were not used. Therefore, this study was effectively conducted for 21 patients (nine male and 12 female, mean age of 35.5 years). Six presented with major-type RAS, while 15 presented with minor types. No patients presented with herpetiform-type RAS.

Biopsy for histological and direct immunofluorescence examination was performed for four patients in order
Table 2 - Characteristics of the patients $(n=21)$

\begin{tabular}{lccc}
\hline Characteristics & Male & Female & Total \\
\hline Age range (mean) years & $10-58(29.9)$ & $13-78(41.9)$ & $10-78(35.5)$ \\
Caucasian & 9 & 10 & 19 \\
Non-caucasian & 0 & 2 & 2 \\
Familial history of RAS & 4 & 7 & 11 \\
Lesions per cycle & & & \\
1 & 0 & 0 & 0 \\
2 to 3 & 2 & 4 & 6 \\
$>3$ & 7 & 8 & 15 \\
Healing time & & & \\
7 to 10 days & 4 & 10 & 14 \\
11 to 20 days & 0 & 0 & 0 \\
21 to 30 days & 5 & 2 & 7 \\
Type of ulcer & & & \\
Major & 5 & 1 & 6 \\
Minor & 4 & 11 & 15 \\
Herpetiform & 0 & 0 & 0 \\
Systemic diseases & & & \\
Yes & 0 & 3 & 3 \\
No & 9 & 9 & 18 \\
Medications & & & \\
Anti-hypertensives & 0 & 2 & 2 \\
Cardiac drugs & 0 & 1 & 1 \\
Thyroid drugs & 0 & 1 & \\
\hline
\end{tabular}

$\mathrm{n}=$ number of patients

to exclude other diseases. Both exams provided nonspecific findings: the histological features consisted predominantly of lymphocytes and neutrophils, and the results of the immunofluorescence technique were nonreactive in all cases. Three female patients presented with systemic diseases: two were hypertensive and one had hypothyroidism. Only one patient was a smoker. The characteristics of the patients are shown in table 2 .

Initial drug assignments are shown in table 3. Eleven patients were switched to alternative drugs. At the end of this trial, the percentage of each drug evaluated was higher than at its initial assignment, as shown in table 4, which summarizes the efficacy of all drugs tested. The most efficient and best-tolerated drug was thalidomide, since patients who used this drug showed an excellent

Table 3 - Drug assignments $(\mathrm{n}=21)$

\begin{tabular}{|c|c|c|c|c|c|}
\hline \multirow[t]{2}{*}{ Drugs } & \multirow{2}{*}{$\begin{array}{c}\text { Initial } \\
\text { Assignments }\end{array}$} & \multicolumn{4}{|c|}{ Switched to } \\
\hline & & Thalidomide & Dapsone & Colchicine & Pentoxifylline \\
\hline Thalidomide & 7 & - & 1 & 1 & - \\
\hline Dapsone & 8 & - & - & 3 & - \\
\hline Colchicine & 5 & 1 & - & - & 4 \\
\hline Pentoxifylline & 1 & - & - & 1 & - \\
\hline
\end{tabular}

$\mathrm{n}=$ number of patients 
Table 4 - Efficacy of the drugs prescribed in the trial

\begin{tabular}{lcccccc}
\hline Drugs & $\begin{array}{c}\text { Initial assignments } \\
(\mathrm{n}=21)\end{array}$ & $\begin{array}{c}\text { Total assignments } \\
\text { throughout trial }\end{array}$ & Excellent & Moderate & Mild & No Response \\
\cline { 4 - 8 } & & 8 & 7 & 0 & 0 & 1 \\
Thalidomide & 8 & 9 & 5 & 3 & 0 & 1 \\
Dapsone & 5 & 10 & 4 & 4 & 1 & 1 \\
Colchicine & 1 & 5 & 1 & 2 & 0 & 2 \\
Pentoxifylline & 8 & &
\end{tabular}

$\mathrm{n}=$ number of patients

Table 5 - Side effects associated with the drugs

\begin{tabular}{|c|c|c|c|c|}
\hline & Thalidomide & Dapsone & Colchicine & Pentoxifylline \\
\hline Assignments & 8 & 9 & 10 & 5 \\
\hline $\begin{array}{l}\text { Period of administration } \\
\text { (mean) }\end{array}$ & $\begin{array}{l}3 \text { to } 6 \text { months } \\
\text { (4.8 months) }\end{array}$ & $\begin{array}{l}1 \text { to } 6 \text { months } \\
\text { (4.2 months) }\end{array}$ & $\begin{array}{l}2 \text { to } 6 \text { months } \\
\text { (4.8 months) }\end{array}$ & $\begin{array}{l}1 \text { to } 6 \text { months } \\
\text { (3.0 months) }\end{array}$ \\
\hline Severe side effects & 0 & $3(33.3 \%)$ & 0 & 0 \\
\hline Moderate side effects & 0 & $3(33.3 \%)$ & 0 & $1(83.3 \%)$ \\
\hline Mild side effects & $1(12.5 \%)$ & 0 & $3(30 \%)$ & 0 \\
\hline No side effects & $7(87.5 \%)$ & $3(33.3 \%)$ & $7(70 \%)$ & $4(16.7 \%)$ \\
\hline
\end{tabular}

response to therapy and did not develop any significant side effects. Dapsone, despite presenting good results, had to be discontinued for most of the patients (six out of nine) due to its adverse side effects. Colchicine and pentoxifylline also provided good results with few side effects; nevertheless, pentoxifylline was used by only five patients due to its financial cost. The side effects experienced were categorized into three types: mild - minor gastrointestinal pain and nausea; moderate - dizziness, fatigue, diarrhea, lethargy, and headache; or severe - hemolysis, jaundice, and decreased hemoglobin level. The mean time for use of each drug and the patient side effects are shown in table 5 .

All patients presented relapses after discontinuing medication during the follow-up period. Recurrences occurred from 2 weeks up to 9 months, but most of the patients experienced relapses within a few weeks after the medication was withdrawn.

\section{DISCUSSION}

Treatment of RAS remains a great challenge. Different kinds of treatment have been attempted over the years, yet no specific management has been established as being the most appropriate..$^{18}$ RAS affects patients differently and, in some cases, may cause severe pain and interfere with one's day-today life. The present study dealt with patients suffering from the severe form of RAS, and therefore received systemic medication.
Each of the four drugs compared in this trial was previously tested separately in other studies. ${ }^{18-26}$ The antiinflammatory and immunological properties of these drugs enable them to reduce or prevent the development of RAS. Dapsone appears to inhibit the migration of polymorphonuclear leukocytes (PMNs) by inhibiting lysosomal enzyme activity and interfering with the cellular response to chemotactic stimuli. ${ }^{27,28}$ Colchicine appears to depolymerize the microtubular proteins of inflammatory cells, inhibiting chemotaxis, mobilization, adhesiveness, and lysosomal degranulation ${ }^{11}$. Thalidomide and pentoxifylline present immunomodulatory and antiinflammatory properties, and inhibit the production of tumor necrosis factor alpha $(\mathrm{TNF} \alpha))^{15,18,22,29}$

A corticosteroid (prednisone) was prescribed for all patients at the beginning of the trial in order to dampen the RAS bout in course. This procedure was carried out to obtain a baseline for comparison of the results and to provide relief of symptoms for the patients. The short-term prescription of a corticosteroid $(0.5 \mathrm{mg} / \mathrm{kg} / \mathrm{day} / 1 \mathrm{week}$, followed by half this dosage for another week) did not cause any significant adverse side effects and was valuable in decreasing patient discomfort. This initial course with a systemic corticosteroid was important for halting bouts of RAS and for patients' compliance with the trial.

Thalidomide was the most effective drug tested in this trial. It was administered to a total of eight patients for a time period ranging from 3 to 6 months (mean, 4.8 months). 
It worked very well in most cases, seven (87.5\%) of which resulted in complete remission of RAS; one patient presented no response. The percentage of complete remission obtained for our patients was much higher than that reported by other authors, namely Grinspan et al. ${ }^{24}(34 \%)$ and Revuz et al. ${ }^{20}(48 \%)$, for which the number of patients treated with thalidomide (40 and 73, respectively) was higher than that in the present study. In terms of side effects, Revuz et al. ${ }^{20}$ found a high percentage of adverse side effects with thalidomide use, mainly drowsiness (75\%) and constipation (40\%). Similar side effects were also observed by Bonnetblanc et al. ${ }^{29}(64 \%)$ and Grinspan et al. ${ }^{24}(48 \%)$. In the present study, only one $(12.5 \%)$ patient complained of slight drowsiness during the thalidomide course.

Dapsone was given to a total of nine patients for lengths of time ranging from 1 to 6 months (mean, 4.2 months). Eight of these patients (89\%) showed excellent-to-moderate improvement of their symptoms. This result was somewhat better than the results obtained by Handfield-Jones et al. ${ }^{19}$, who reported improvement in $50 \%$ of their patients using the same protocol and dosage as those used in our trial $(100 \mathrm{mg} /$ day). These authors did not mention side effects with the dapsone use. Dapsone had to be discontinued for six of our patients due to its moderate-to-severe side effects. In three of these patients, anemia, hemolysis, and jaundice occurred early in the course of medication, and the patients did not receive any other systemic medication until they had completely recovered to a healthy clinical condition. Other authors using dapsone for ulcerative mucosal diseases other than RAS have reported similar side effects, although these were less severe and more delayed in their onset. ${ }^{27,30}$

During the course of the trial, colchicine was prescribed to a total of ten patients for a period of time ranging from 2 to 6 months (mean, 4.8 months). Benefits were obtained with colchicine in nine patients $(90 \%)$, among which four experienced complete remission of lesions, four demonstrated moderate improvement, and one experienced only a mild benefit. Efficacy with the use of colchicine for treatment of RAS has also been reported by other authors ${ }^{21,25}$, with improvement ranging from 63 to $71 \%$ of the cases. In one of these studies ${ }^{25}$, patients were followed for as long as 4.7 years and, at the end of that period, $37 \%$ of the patients (without medication) still maintained a better clinical status compared with the period before treatment. Side effects with colchicine have been observed in many studies ${ }^{11,21,31,32}$, and are mainly characterized by gastrointestinal disturbances (e.g., diarrhea and nausea) and paresthesia of the lower extremities. Ruah et al. ${ }^{11}$ reported that the continuous use of colchicine could lead to the development of neuropathy and myopathy. In the present study, three patients complained of some discomfort (diarrhea), which was easily controlled by slightly lowering their dosage.

Pentoxifylline was prescribed for only five patients since the cost of this medication impeded its availability in the present trial. Pentoxifylline was used for a period ranging from 1 to 6 months (mean, 3.0 months). In two patients $(40 \%)$, a moderate improvement was observed (shorter healing time, less severe symptoms, and a lower recurrence rate), while one patient (20\%) presented with complete remission and two others experienced no benefit. These results were in agreement with those of Pizarro et al. ${ }^{22}$ and Chandrasekhar et al. ${ }^{15}$, who obtained excellent-to-satisfactory results with pentoxifylline in 50 to $60 \%$ of their patients. Even better results were obtained by Wahba-Yahva ${ }^{23}$, who reported stable remission in all cases (six patients). Side effects were seldom reported by patients in those studies. In the present study, the patients also presented few side effects, with only one patient manifesting dizziness and nausea and for whom the medication was discontinued.

Regarding the effect of the drugs in preventing RAS recurrences after their withdrawal, dapsone was found to yield the best results. Of the patients who reported benefits with dapsone, three remained ulcer free without medication for up to 9 months. Colchicine apparently did not protect patients against new bouts of RAS after withdrawal, since recurrences occurred within 2 to 3 weeks after drug discontinuation. Similar results were observed with pentoxifylline, as just one patient remained ulcer free for a 5-month period of time after drug withdrawal. As for thalidomide, patients experienced no recurrences for a 2-week to 4-month period after its withdrawal. Of note, three patients who had their th alidomide prescription discontinued during the course of therapy due to a temporary interruption in hospital supply experienced a rapid worsening of their clinical status, which was not improved by any other drug used in this trial. These patients were placed back on thalidomide as soon as it became available and regained the clinical status they presented before the interruption. It is also important to mention that, five of the six patients with major-type RAS, benefited from the treatment protocol with thalidomide, thus reinforcing the effectiveness of this drug for RAS treatment.

\section{CONCLUSION}

The therapeutic methods used in this trial were able to provide significant relief of symptoms for as long as the drug was administered. Relapses of the lesions after withdrawal of the medication, however, were observed during the follow-up period. Thalidomide provided the best results in our trial, but 
this drug presents problems with regard to accessibility, and its use is precluded for fertile women. Dapsone produced benefits, but also resulted in severe adverse side effects.
Pentoxifylline was moderately effective, although it was not properly tested in this trial. Colchicine demonstrated good results and was well tolerated by the patients.

\section{REFERENCES}

1. Scully C, Gorsky M, Lozada-Nur F. The diagnosis and management of recurrent aphthous stomatitis, a consensus approach. J Am Dent Assoc. 2003;134:200-7.

2. Vincent SD, Lilly GE. Clinical, historic, and therapeutic features of aphthous stomatitis. Literature review and open clinical trial employing steroids. Oral Surg Oral Med Oral Pathol. 1992;74:79-86.

3. Natah SS, Konttinen YT, Enattah NS, Ashammakhi N, Shrakey KA, Häyrinen-Immonen R. Recurrent aphthous ulcers today: a review of the growing knowledge. Int J Oral Maxillofac Surg. 2004;33:221-34.

4. Reichart PA. Oral mucosal lesions in a representative cross-sectional study of aging Germans. Community Dent Oral Epidemiol. 2000;28:3908.

5. Kleinman DV, Swango PA, Niessen LC. Epidemiologic studies of oral mucosal conditions - methodologic issues. Community Dent Oral Epidemiol. 1991;19:129-40.

6. Pedersen A, Hougen HP, Kenrad B. T-lymphocyte subsets in oral mucosa of patients with recurrent aphthous ulceration. J Oral Pathol Med. 1992;21:176-80.

7. Casiglia JM. Recurrent aphthous stomatitis: Etiology, diagnosis, and treatment. Gen Dent. 2002;50:157-66.

8. Porter SR, Scully C. Aphthous stomatitis - an overview of aetiopathogenesis and management. Clin Exp Dermatol. 1991;16:23543.

9. Woo SB, Sonis ST. Recurrent aphthous ulcers: A review of diagnosis and treatment. J Am Dent Assoc. 1996;27:1202-13.

10. Field EA, Allan RB. Review article: oral ulceration - aetiopathogenesis, clinical diagnosis and management in the gastrointestinal clinic. Aliment Pharmacol Ther. 2003;18:949-62.

11. Ruah CB, Stram JR, Chasin WD. Treatment of severe recurrent aphthous stomatitis with colchicine. Arch Otolaryngol Head Neck Surg. 1988;114:671-5.

12. Eversole LR. Immunopathology of oral mucosal ulcerative, desquamative, and bullous diseases. Selective review of the literature. Oral Surg Oral Med Oral Pathol. 1994;77:555-71.

13. Ship JA, Chavez EM, Doerr PA, Henson BS, Sarmadi M. Recurrent aphthous stomatitis. Quintessence Int. 2000;31:95-112.

14. Ship JA. Recurrent aphthous stomatitis: An update. Oral Surg Oral Med Oral Pathol Oral Radiol Endod. 1996;81:141-7.

15. Chandrasekhar J, Liem AA, Cox, NH, Paterson AW. Oxypentoxifylline in the management of recurrent aphthous oral ulcers. An open clinical trial. Oral Surg Oral Med Oral Pathol Oral Radiol Endod. 1999;87:5647.

16. Rodu B, Mattingly G. Oral mucosal ulcers: diagnosis and management. J Am Dent Assoc. 1992;123:83-6.
17. Barrons RW. Treatment strategies for recurrent oral aphthous ulcers. Am J Health Syst Pharm. 2001;58:41-50.

18. Jurge S, Kuffer R, Scully C, Porter SR. Recurrent aphthous stomatitis. Oral Dis. 2006;12:1-21.

19. Handfield-Jones S, Allen BR, Littlewood SM. Dapsone use with oralgenital ulcers. Br J Dermatol. 1985;113:501.

20. Revuz J, Guillaume JC, Janier M, Hans P, Marchand C, Souteyrand $\mathrm{P}$, et al. Crossover study of thalidomide vs placebo in severe recurrent aphthous stomatitis. Arch Dermatol. 1990;126:923-7.

21. Katz J, Langevitz P, Shemer J, Barak S, Livneh A. Prevention of recurrent aphthous stomatitis with colchicine: An open trial. J Am Acad Dermatol. 1994;31:459-61.

22. Pizarro A, Navarro A, Fonseca E, Vidaurrazaga C, Herranz P. Treatment of recurrent aphthous stomatitis with pentoxifylline. Br J. Dermatol. 1995; 133:659-60.

23. Wahba-Yahav AV. Pentoxifylline in intractable recurrent aphthous stomatitis: an open trial. J Am Acad Dermatol. 1995;33:680-2.

24. Grinspan D, Blanco GF, Agüero S. Treatment of aphthae with thalidomide. J Am Acad Dermatol. 1989;20:1060-3.

25. Fontes V, Machet L, Huttenberger B, Lorette G, Vaillant L. [Recurrent aphthous stomatitis: treatment with colchicine. An open trial of 54 cases]. Ann Dermatol Venereol. 2002;129:1365-9.

26. Altinor S, Öztürkcan S, Hah MM. The effects of colchicine on neutrophil function in subjects with recurrent aphthous stomatitis. J Eur Acad Dermatol Venearol. 2003; 17:469-70.

27. Ciarrocca KN, Greenberg MS. A retrospective study of the management of oral mucous membrane pemphigoid with dapsone. Oral Surg Oral Med Oral Pathol Oral Radiol Endod. 1999;88:159-63.

28. Modschiedler K, Weller M, Wörl P, Von Den Driesch P. Dapsone and colchicine inhibit adhesion of neutrophilic granulocytes to epidermal sections. Arch Dermatol Res. 2000;292:32-6.

29. Bonnetblanc J-M, Royer C, Bedane C. Thalidomide and recurrent aphthous stomatitis: A follow-up study. Dermatology. 1996;193:3213.

30. Ahmed AR, Bradley SK, Rogers III RS. Cicatricial pemphigoid. J Am Acad Dermatol. 1991;24:987-1001.

31. Miyachi Y, Taniguchi S, Ozaki M, Horio T. Colchicine in the treatment of the cutaneous manifestations of Behçet's disease. Br J. Dermatol. 1981;104:67-9.

32. Nicolás JLL, Íñiguez JCM. Tratamiento de la estomatitis aftosa recidivante: Revisón bibliográfica. Rev Clin Esp. 1998;198:234-6. 